\section{Modified marching-in-place test for interlimb coordination as a screening tool for fall risk in older adults}

\author{
Sibadiuty Mohapatra, BOT, MOT, Swati Sweta Das, BOT, MOT, Sebestina Anita \\ Dsouza, MOT, PhD
}

\begin{abstract}
Background. Interlimb coordination is an essential component of postural stability. The marching-in-place (MIP) test is a practical tool that requires little space and time and no equipment. However, its usefulness to assess fall risk in older adults is unknown. The present study aimed to determine the association of the MIP test results with fall risk and to establish its psychometric properties as a screening tool for fall risk in older adults.
\end{abstract}

Methods: The study involved two phases. Phase I was a pilot in 15 older adults. The MIP test was evaluated and modified to improve its feasibility as a screening tool. Phase II was a cross-sectional study involving 288 community-dwelling older adults. Participants were assessed for fall history and balance-related fall risk (using the timed up and go test and the four square step test) and the modified MIP test.

Results: Impaired interlimb coordination in the modified MIP test was associated with fall risk $(p<0.001)$ and a history of fall $(p=0.001)$. The modified MIP test had a sensitivity of $96.83 \%$, specificity of $46.22 \%$, positive predictive value of $33.52 \%$, and negative predictive value of 98.11\%.

Conclusion: The modified MIP test results are associated with fall risk and fall history. Its psychometric properties support its use as an inexpensive and feasible screening tool for fall risk in older adults.

Key words: Accidental falls; Aged; Coordination complexes; Predictive value of tests; Risk factors; Sensitivity and specificity

\section{ORIGINAL ARTICLE}

Department of Occupational Therapy, Centre for Studies on Healthy Aging, Manipal College of Health Professions, Manipal Academy of Higher Education, Manipal, Karnataka, India

\section{INTRODUCTION}

Interlimb coordination involves spatiotemporal organisation of multiple limbs or segments and is an essential aspect of daily activities including walking. ${ }^{1,2}$ Interlimb coordination in normal gait involves automatic swinging of the arms by pairing upper and lower limb movements with integer ratio frequencies. Although initially considered as a remnant feature of human evolution from quadruped to bipedal locomotion, these automatic coordinated movements suggest involvement of the motor cortex, ${ }^{3,4}$ and functional neural coupling between arms and legs during walking, ${ }^{5}$ and are critical for stable walking and for reducing energy expenditure. ${ }^{6,7}$

Interlimb coordination deteriorates with age and may be associated with fall risk in older adults. Training to improve interlimb coordination is suggested to enhance gait stability even in high-functioning older adults. ${ }^{8}$ Considering the association of interlimb coordination with fall risk, assessing interlimb coordination in clinical settings is valuable in fall prevention programmes. Currently, assessment of interlimb coordination is predominantly laboratory- 
based using a treadmill, which is highly contextspecific and could be physically and cognitively demanding for people with mobility impairments. ${ }^{4,8}$ These assessments require special equipment and space, making it difficult to perform in clinical settings, especially in resource-constrained areas such as India.

The marching-in-place (MIP) test was developed to assess interlimb coordination in clinical settings. ${ }^{9}$ The test is based on an assumption that MIP is similar to walking with respect to interlimb coordination. Most people have experience in marching since childhood. MIP would thus be easy for them to understand and would not require much space, making it feasible for clinical settings. Participants are asked to MIP for 1 minute. Normal interlimb coordination refers to marching with synchronous contralateral movement patterns of limbs without losing balance. Partially impaired interlimb coordination refers to marching with inconsistent, asynchronous or irregular movement patterns of contralateral limbs but able to maintain balance. Impaired interlimb coordination refers to the inability to maintain balance. Performance on the MIP test deteriorated with increasing age, and men performed better than women. ${ }^{9}$

The prevalence of falls among Indian older adults was reported to be $14 \%$ to $35 \%$; fall risk screening and prevention is thus of utmost importance. ${ }^{10}$ For use in busy hospital out-patient departments, primary healthcare centres, or community settings, screening tools should be inexpensive and require little time and space. The ability of the MIP test to assess fall risk is unknown. Therefore, the present study aims to determine the association of the MIP test results with fall history and fall risk, and the usefulness of MIP as a screening tool to assess fall risk in older adults.

\section{METHODS}

This cross-sectional observational study was approved by the institutional ethics committee (reference: IEC 742/2016). Data were collected between November 2016 and April 2017. The study involved two phases.

\section{Phase 1}

To determine the feasibility of the MIP as a screening tool, the MIP performance of 15 older adults was recorded on video and participants' feedback collected. Investigators of the present study felt that some modifications were required to improve its feasibility as a screening tool, and the authors of the original test consented for the modifications. In the modified MIP test, the duration of each trial was reduced to $30 \mathrm{~s}$, as some participants reported fatigue. This also reduced the overall time required for the test without affecting rating. In addition, the rating criteria was modified to include two categories only (normal and impaired interlimb coordination). Thus, participants who marched in place for $30 \mathrm{~s}$ with synchronous contralateral movement patterns of the limbs without losing balance were rated as normal interlimb coordination and as negative on the screening test. Participants who did not meet this description were rated as impaired interlimb coordination and as positive on the screening test.

\section{Phase II}

To determine the psychometric properties of the modified MIP as a screening tool, a cross-sectional study was conducted in the Udupi district, Karnataka, India, and the Khurda district, Odisha, India. In total, 387 community-dwelling older adults were recruited by convenience sampling from community parks, temples, health camps, community hospitals, and private homes. Of them, 11 refused to participate owing to video recording and 88 consented but did not meet the study criteria and were excluded. The remaining 288 older adults met the study criteria.

The inclusion criteria included: age 65 to 80 years, walking independently with or without walking aids, and consent for recording their performance on video. The exclusion criteria included: having any acute medical illness, current lower or upper limb injury, walking with physical assistance by another person, fear of fall, any orthopaedic conditions or moderate to severe neurological illness such as stroke and Parkinson disease, a score of $<6 / 18$ or $<20 / 60$ on the Snellen visual screening test with visual aids, and difficulty in understanding and following instructions owing to cognitive impairments as suggested by a score of $<26$ on the Montreal Cognitive Assessment. ${ }^{11}$

The modified MIP test was benchmarked against the gold standard tests that assess similar dimension of fall risk (ie, balance). The timed up and go test (TUG) is one of the most commonly used tests to 
evaluate functional mobility, but it has limited ability to predict falls in community-dwelling elderly and necessitates combining with other standardised measures to improve its predictive value..$^{12-14}$ Therefore, TUG was combined with the four square step test (FSST) for dynamic balance to determine the risk of fall secondary to balance-related impairments.

Participants performed the TUG and the FSST, and the time taken to perform the tests were noted. They then performed the modified MIP test, with their performance recorded on video. Participants were categorised as 'no risk' if they were below the cut-offs for both TUG and FSST. Participants who did not meet the cut-offs of one (either TUG or FSST) or both tests were considered as 'at risk'.

For the TUG, a chair with armrests was placed with a line marked at a distance of 10 feet from the chair. Participants were instructed to stand up from the chair, walk towards the line at a normal pace, turn, return to the chair, and sit down. Two trials were recorded after one practice trial. The best of the two trials was recorded as the final score. Participants were allowed to wear their regular footwear and to use the arm rest as support while getting up and sitting down. The cut-off score for TUG is $12 \mathrm{~s} .{ }^{15}$ The TUG has sensitivity of $87 \%$ and specificity of $87 \%$ to identify community dwelling older adults who have risk of fall. ${ }^{16}$

For the FSST, four sticks, each $90 \mathrm{~cm}$ long and $2.5 \mathrm{~cm}$ thick, were placed flat on the floor in a cross shape, forming 4 squares. The participant stood in square number 1 facing square number 2 and stepped as fast as possible into each square in the following sequence of square number: $2,3,4,1,4$, 3,2 , and 1 . This sequence required the participant to step forwards, sidestep right, step backwards, and sidestep left. The time taken to complete the sequence was recorded. Two trials of the test were completed with the best time taken as the score. Using the cut-off of $15 \mathrm{~s}$, the FSST is $85 \%$ sensitive and $88 \%$ specific in determining risk of fall. ${ }^{17}$

In the modified MIP test, participants were first instructed to march in place for $30 \mathrm{~s}$. Participants were given one practice trial and then performed three test trials, which were recorded. Instructions were repeated with demonstrations if participants had difficulties in performing the test, such as moving out of place, looking down at their feet while marching, or marching without arm movements. The initial two test trials enabled participants to familiarise themselves with the test. The third test trial was considered as the best performance and was used for analysis. The performance was rated as normal (negative result) or impaired (positive result) interlimb coordination. Adequate rest was provided between trials to avoid fatigue. The investigator stood on the left side to assist the participant in case of any imbalance. The entire test took around 5 minutes. The video recordings were used as an adjunct to support movement analysis and scoring only. The video camera was mounted on a tripod at $12 \mathrm{~m}$ from the right side of the participant.

SPSS (Windows version 20; IBM Corp, Armonk [NY], US) was used for data analysis. Chi squared test was used to determine the association of interlimb coordination with falls and fall risk in communitydwelling older adults. The level of significance was set at $\mathrm{p}<0.05$. Specificity, sensitivity, and predictive values for the MIP test were determined.

\section{RESULTS}

288 (160 male and 128 female) older adults (mean age, $70.08 \pm 4.16$ years) were included. Of these, four $(1.7 \%)$ used mobility aids and $36(12.5 \%)$ had a history of fall in the past year (26 with one fall event, eight with two fall events, and two with three fall events).

Impaired interlimb coordination in the MIP test was associated with a history of fall and fall risk. More participants with a history of fall than those with no history of fall had impaired interlimb coordination $(80.06 \%$ [26/29] vs $60.71 \%$ [153/252], $\mathrm{p}=0.001)$. Similarly, more participants with risk of fall than those with no risk of fall had impaired interlimb coordination $(96.82 \%$ [61/63] vs 53.77\% [121/225], $\mathrm{p}<0.001)$ [TABLE $\mathbf{1}$.

The sensitivity value indicated that of the 63 participants identified as 'at risk' by TUG and FSST, the modified MIP correctly detected $96.8 \%$ as having fall risk (positive result). The specificity value indicated that modified MIP accurately identified $46 \%$ (negative result) of the 225 participants who were identified as no risk by TUG and FSST. The positive predictive value indicated that an older adult 
with impaired interlimb coordination (positive result on modified MIP) will have $33.52 \%$ probability of having the risk of fall. The negative predictive value indicated that an older adult with normal interlimb coordination (negative result on modified MIP) will have $98.11 \%$ probability of not having the risk of fall (TABLE 2).

\section{DISCUSSION}

In the present study, MIP results were associated with history of fall and balance-related fall risk. The findings suggest an association between interlimb coordination and fall risk, and further validate suggestions by Krasovsky et al. ${ }^{8}$

The present study demonstrated that the modified MIP test has high sensitivity and low specificity. The high sensitivity permits people with normal interlimb coordination in the modified MIP (negative result) to be confidently regarded as not having fall risk and could be ruled out. However, the moderate specificity suggests a fairly high rate of false positives of those identified as having impaired interlimb coordination on the modified MIP (positive result). They may need further follow-up assessment to confirm their fallrisk. This high sensitivity and moderate specificity could be considered as acceptable for a screening tool as it is very important not to miss cases. ${ }^{18}$

Sensitivity and specificity are test psychometrics and indicate the effectiveness of a screening tool based on their comparison with a gold standard. These values would be an important consideration when selecting or choosing a screening tool. However, they are not very useful in clinical settings when making decisions whether the person being screened has the condition or not. In such conditions, predictive values are more useful. ${ }^{18}$

The high negative predictive value of the modified MIP test is very useful as it minimises the false negatives during screening. ${ }^{18}$ As the positive predictive value is moderate, those who tested positive may need to be tested further with more reliable and valid assessments to confirm their fall risk. The moderate positive predictive value of the modified MIP test could be considered as acceptable as the follow-up tests for fall risk assessment are not very expensive and stressful for older adults. Also, the false positive screening outcomes may be permissible if no harm is likely to be done to them in protecting against fall risk, even if it is not present. For example, older adults may be told they have fall risk based on their performance on the modified MIP test, even though they do not have any fall risk. They would be advised on fall prevention such as fall education, balance training, or environmental modifications. They would benefit if they adopt the advised strategies.

In developing countries such as India, fall risk

TABLE 1

Association of interlimb coordination with history of fall and risk of fall

\begin{tabular}{|c|c|c|c|c|c|c|}
\hline \multirow[t]{2}{*}{$\begin{array}{l}\text { Interlimb } \\
\text { coordination }\end{array}$} & \multicolumn{2}{|c|}{ History of fall } & \multirow[t]{2}{*}{$\mathrm{p}$ Value } & \multicolumn{2}{|c|}{$\begin{array}{l}\text { Risk of fall (timed up and go test and } \\
\text { four square step test) }\end{array}$} & \multirow[t]{2}{*}{$\mathrm{p}$ Value } \\
\hline & Present $(n=36)^{\star}$ & Absent $(n=252)^{\star}$ & & At-risk $(n=63)^{\star}$ & No-risk $(n=225)^{\star}$ & \\
\hline Impaired (positive) & $29(80.06)$ & $153(60.71)$ & 0.001 & $61(96.82)$ & $121(53.77)$ & $<0.001$ \\
\hline Normal (negative) & 07 (19.4) & 99 (39.28) & & $2(3.17)$ & 104 (46.22) & \\
\hline
\end{tabular}

* Data are presented as No. (\%) of participants

TABLE 2

Psychometric properties of modified marching-in-place test

\begin{tabular}{lc}
\hline Modified marching-in-place test & Odds ratio (95\% confidence interval) \\
\hline Sensitivity & $96.82 \%(89.00 \%-99.61 \%)$ \\
Specificity & $46.22 \%(39.57 \%-52.97 \%)$ \\
Positive predictive value & $33.52 \%(30.70 \%-36.45 \%)$ \\
Negative predictive value & $98.11 \%(92.96 \%-99.51 \%)$ \\
\hline
\end{tabular}


assessment usually occurs in busy and crowded outpatient settings in a hospital or in community-based health camps that usually have space constraints. In such settings, well established tests such as the TUG, Berg Balance Scale, or FSST are difficult to use as they require designated test area, arrangement of test materials, more time and instructions. The modified MIP test can reduce the time for fall risk assessment and allow evaluation of more people. Older adults identified with impaired interlimb coordination in the modified MIP test can be called for a detailed fall-risk assessment with well-established tests on subsequent visits or appointments.

The present study has some limitations. Recall bias may be present regarding history of falls in the past year. Some participants were uncomfortable with the video recordings and that may have influenced their performance. As with any test, the modified MIP may also have random measurement errors secondary to participant-related factors such as nervousness, lack of comprehension, lack of confidence, or distraction during the test. This has been addressed by allowing the participant to practice before measurement in the final trial. In addition, the examiner may also contribute to the measurement error, including variations among examiners or clinicians with respect to adherence to the procedure, instructions, and interpretation of the rating criteria. The rating criteria is based on movement quality. Thus, the examiner's observational skills and experience may influence test interpretation. ${ }^{19}$ Therefore, further studies are required to determine inter-rater and intra-rater reliability of the modified MIP test. The modified MIP test can be used by community health workers for fall-risk screening during home visits or at primary healthcare centres. Future studies are warranted to explore this aspect.

\section{CONCLUSION}

Interlimb coordination is associated with history of fall and balance-related fall risk among older adults in the community. Psychometric properties of the modified MIP test support its use as a screening tool for balance-related fall risk. The study has implications for community-based or population-based fall risk screening and fall prevention programmes for older adults.

\section{REFERENCES}

1. Heuninckx S, Debaere F, Wenderoth N, Verchueren S, Swinnen SP. Ipsilateral coordination deficits and central processing requirements associated with coordination as a function of aging. J Gerontol B Psychol Sci Soc Sci 2004;59:225-32. Crossref

2. Fujiyama H. Age-related Changes in Interlimb Coordination. Australia, University of Tasmania; 2009. Available from: https:// eprints.utas.edu.au/9679/1/Fujiyama_Thesis_2009.pdf. Accessed 27 March 2020.

3. Barthelemy D, Nielsen JB. Corticospinal contribution to arm muscle activity during human walking. J Physiol 2010;588:967-79. Crossref

4. Bruijn SM, Meijer OG, Beek PJ, van Dieen JH. The effects of arm swing on human gait stability. J Exp Biol 2010;213:3945-52. Crossref

5. Zehr EP, Hundza SR, Vasudevan EV. The quadrupedal nature of human bipedal locomotion. Exerc Sport Sci Rev 2009;37:1028. Crossref

6. Courtine G, Schieppati M. Tuning of a basic coordination pattern constructs straight-ahead and curved walking in humans. J Neurophysiol 2004;91:1524-35. Crossref

7. Collins SH, Adamczyk PG, Kuo AD. Dynamic arm swinging in human walking. Proc Biol Sci 2009;276:3679-88. Crossref

8. Krasovsky T, Banina MC, Hacmon R, Feldman AG, Lamontagne A, Levin MF. Stability of gait and interlimb coordination in older adults. J Neurophysiol 2012;107:2560-9. Crossref

9. Das SS, Dsouza SA. Development of a clinical test to assess interlimb coordination in older adults: a preliminary study. Manipal: Manipal Academy of Higher Education; 2015.

10. Dsouza SA, Rajashekar B, Dsouza HS, Kumar KB. Falls in older adults: a barrier to active ageing in India. Asian J Gerontol Geriatr 2014;9:33-40.

11. Doerflinger DMC. Mental Status Assessment in Older Adults: Montreal Cognitive Assessment: MOCA Version 7.1. New York: New York University, The Hartford Institute for Geriatric Nursing; 2012. Available from consultgeri.org/try- this/generalassessments /issue-3.2.pdf. Accessed 27 March 2020.

12. Barry E, Galvin R, Keogh C, Horgan F, Fahey T. Is the Timed $\mathrm{Up}$ and Go test a useful predictor of risk of falls in community dwelling older adults: a systematic review and meta-analysis. BMC Geriatr 2014;14:14. Crossref

13. Tiedemann A, Shimada H, Sherrington C, Murray S, Lord S. The comparative ability of eight functional mobility tests for predicting falls in community-dwelling older people. Age Ageing 2008;37:430-5. Crossref

14. Lusardi MM, Fritz S, Middleton A, Allison L, Wingood M, Phillips E, et al. Determining risk of falls in community dwelling older adults: a systematic review and meta-analysis using posttest probability. J Geriatr Phys Ther 2017;40:1-36 crossref

15. Bischoff HA, Stahelin HB, Monsch AU, Iversen MD, Weyh A, von Dechend MV, et al. Identifying a cut-off point for normal mobility: a comparison of the timed 'up and go' test in community-dwelling and institutionalised elderly women. Age Ageing 2003;32:315-20. Crossref

16. Shumway-Cook A, Brauer S, Woollacott M. Predicting the probability for falls in community-dwelling older adults using the Timed UP \& Go Test. Phys Ther 2000;80:896-903. Crossref

17. Dite W, Temple VA. A clinical test of stepping and change of direction to identify multiple falling older adults. Arch Phys Med Rehabil 2002;83:1566-71. Crossref

18. Trevethan R. Sensitivity, specificity, and predictive values: foundations, pliabilities, and pitfalls in research and practice. Front Public Health 2017;5:307. Crossref

19. Bialocerkowski AE, Bragge P. Measurement error and reliability testing: application to rehabilitation. Int $\mathrm{J}$ Ther Rehabil 2008;15:422-7. Crossref 